\title{
Professor John H. S. Lee: The Detonation Phenomenon
}

\author{
Cambridge University Press, 2008, 400 pp., ISBN: 9780521897235, \$99.00
}

Published online: 24 March 2009

(c) The Author(s) 2009. This article is published with open access at Springerlink.com

The book "The Detonation Phenomenon" by Professor John H. S. Lee is intended for engineers and graduate students with backgrounds in thermodynamics and fluid dynamics. It fulfills that mission perfectly. The book is an excellent, thorough review of the basic experimental and theoretical aspects of gas phase detonation. Gas phase detonation has frequently been studied, because its three-dimensional shock wave structure can be photographed, the pressure it produces is not extreme, as opposed to that of a solid or liquid explosive, and the chemical reaction rates that control its propagation can be measured in shock tubes. Professor Lee has assembled a wonderful combination of fantastic photographs from all over the world plus basic theoretical explanations of the various phenomena involved in gas phase detonation. The book has a mechanical engineering slant, emphasizing the structures of shock and detonation waves and their interactions with surrounding materials. The book covers the discovery of detonation with pictures of the scientists who uncovered the basic properties of detonation waves. The first three chapters and part of Chapter 4 contain necessary experimental and theoretical background found in other books, but this information is presented in a very clear concise manner. Each chapter ends with closing remarks, which often contain a list of unsolved problems. The bibliographies at the end of each chapter contain most of the relevant references.

The remaining chapters of "The Detonation Phenomena" are unique and brilliantly presented. Chapter 5 is a numerical analysis of unstable detonation that provides an excellent summary of several complex topics. Professor Lee systematically discusses: linear stability analysis; asymptotic modeling; direct computer simulations; and chemical effects on the stability of detonation waves. He ends Chapter 5 with its main message that theory and numerical modeling support that the experimental fact that all detonation waves are unstable and exhibit multi-dimensional wave structures.

Chapter 6 is entitled "Unstable Detonations: Experimental Observations." It begins with the 1926 discovery of "spinning detonation" and presents photographs and explanations of all of the detonation flows discovered thus far. This chapter is by far the best collection of famous photographs from many authors of detonation wave structures. Many cases of multi-headed detonation waves and the triple shock intersection points that control their propagation are illustrated. The effects of various geometries on the "cellular structures" produced by the interactions of these "triple points" are covered more completely than in other books on gaseous detonation. Chapter 6 ends with a basic discussion of how chemical energy release controls detonation wave cell size.

Chapter 7 discusses the influence of boundary conditions on gaseous detonation wave propagation. The influences of rough versus smooth walls (turbulent flow) on detonation velocity and cellular structures are important engineering concerns that are covered very well in Chapter 7. An imaginative experiment developed by Professor Lee's group was to pass a detonation wave over an acoustically absorbing material. When the triple shock intersection points of a detonation waves interacted with this material, the cellular structure of the detonation was destroyed. The detonation wave exited that section of tube as a planar shock wave followed by a deflagration wave. This experiment proved that the cellular structure was essential to gas phase detonation

C. M. Tarver

Energetic Materials Center, Lawrence Livermore National Laboratory, University of California, Livermore, CA, USA

E-mail: tarver1@1lnl.gov 
wave propagation. The last section of Chapter 7 discusses another practical aspect of gas phase detonation: limiting fuel—oxidizer — dilutent concentrations required for sustained detonation wave propagation.

Chapter 8 discusses deflagration-to-detonation transitions, which are essential to understand for intentional initiation of detonation and for prevention of accidental detonation during combustion. A discussion of deflagration is followed by a full description of the important features of the transition. Photographic examples of all these features are provided. The various mechanisms by which flames (deflagrations) are accelerated and amplify pressure waves into shock waves are explained with numerous illustrations. The onset of detonation after turbulent flame acceleration and shock wave formation is the next subject. The classic photographs of Urtiew and Oppenheim illuminating several deflagration-to-detonation transition flows are shown. Professor Lee introduces the SWACER mechanism by which chemical energy release is synchronized with the forming shock wave(s) that cause detonation. After the experimental deflagration-to-detonation transition results are presented, an analytical criterion for the process to occur is discussed in terms of turbulent flow and amplification of transverse pressure waves. This chapter ends with a summary of the deflagration-to-detonation transition process and identifies areas of future research.

Finally, Chapter 9 is concerned with various methods of direct initiation of detonation that do not necessarily depend on deflagration-to-detonation transition. These methods include initiation by a blast wave, by a shock or detonation wave, or by a jet of combustion products. As for the other phenomena discussed in this book, direct initiation methods are thoroughly examined experimentally and theoretically. Practical effects, such as the critical tube diameter for sustained detonation propagation and effects of geometry and chemical composition changes on direct initiation, are included. The author concludes the book with a discussion of the importance of the SWACER mechanism in direct initiation of self-sustaining gas phase detonation wave propagation.

In conclusion, the new book "The Detonation Phenomenon" is an excellent overall description of the processes involved in gas phase detonation. It is a product of over 40 years of unique research by Professor Lee and his group. It provides an excellent textbook for a graduate level engineering course on gaseous reactive flows. It is also required reading for those interested in detonation, deflagration, and other reactive flows. Additional important detonation phenomena not covered in this book include: the non-equilibrium processes that precede and follow chemical energy release behind individual shock waves and the physical mechanism by which chemical energy in the product gases amplifies these shocks and maintains the average constant detonation velocity.

Open Access This article is distributed under the terms of the Creative Commons Attribution Noncommercial License which permits any noncommercial use, distribution, and reproduction in any medium, provided the original author(s) and source are credited. 